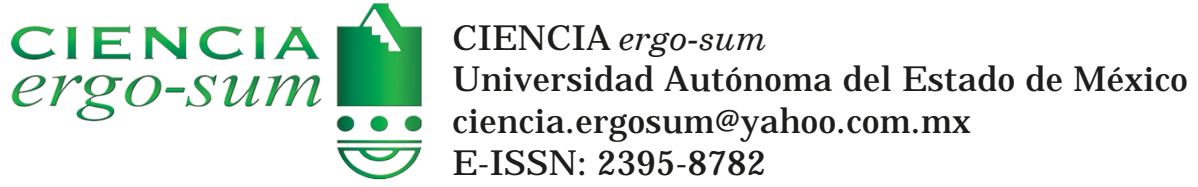

\title{
Nivel de mecanización agrícola en el municipio de Zinacantepec, Estado de México
}

Hernández Ávila, J esús; Gutiérrez Rodríguez, Francisco; González Huerta, Andrés; Bailón Sáenz, Hilda Consuelo Nivel de mecanización agrícola en el municipio de Zinacantepec, Estado de México

CIENCIA ergo-sum, vol. 27, núm. 1, marzo-junio 2020|e75

Universidad Autónoma del Estado de México, México

Esta obra está bajo una Licencia Creative Commons Atribución-NoComercial-SinDerivar 4.0 Internacional .

Hernández Ávila, J ., Gutiérrez Rodríguez, F., González Huerta, A. y Bailón Sáenz, H. C. (2020). Nivel de mecanización agrícola en el municipio de Zinacantepec, Estado de México. CIENCIA ergo-sum, 27(1). https://doi .org/10.30878/ces. v27nla7 


\title{
Nivel de mecanización agrícola en el municipio de Zinacantepec, Estado de México
}

Agricultural mechanization level in the municipality of Zinacantepec, State of Mexico

\author{
Jesús Hernández Avila \\ Universidad Autónoma del Estado de México, México \\ jha333@gmail.com \\ Francisco Gutiérrez Rodríguez \\ Universidad Autónoma del Estado de México, México \\ fgrfca@hotmail.com \\ Andrés González Huerta \\ Universidad Autónoma del Estado de México, México \\ agonzalezh@uaemex.mx \\ Hilda Consuelo Bailón Sáenz \\ Secretaría de Desarrollo Agropecuario del Gobierno del Estado de México, México \\ hildabailon61@yahoo.com
}

Recepción: 20 de agosto de 2018

Aprobación: 14 de marzo de 2019

\section{RESUMEN}

Se lleva a cabo una investigación en el municipio de Zinacantepec, Estado de México, por medio de un estudio exploratorio, descriptivo no experimental, con el objetivo de conocer la situación actual del nivel de mecanización de los tractores y las máquinas agrícolas que poseen los productores agropecuarios y así identificar índices técnicos para optimizarlos en aras de una productividad eficiente. El promedio de edad que tienen los tractores es de 7.73 años, su concentración se localiza en $77.38 \%$ en superficies menores a 10 ha, lo cual indica una subutilización, aunado a un programa de mantenimiento no adecuado que conlleva al deterioro prematuro del tractor.

PALABRAS ClaVES: máquinas agrícolas, moto-horas, potencia por hectárea, tractores.

\begin{abstract}
The investigation was carried out in the municipality of Zinacantepec, State of Mexico, through an exploratory, descriptive, nonexperimental study, The main objective is to know the current situation of the level of mechanization of the tractors and agricultural machines that agricultural producers have, to identify technical indexes, in order to optimize them for the sake of efficient productivity. The average age of tractors is 7.73 years old, its concentration is located at $77.38 \%$ in areas smaller than 10 ha; which indicates underutilization, joined with an inadequate maintenance program; which leads to premature tractor danger.
\end{abstract}

KEYWORDS: agricultural machines, moto-hours, power per hectare, tractors.

\section{INTRODUCCIÓN}

La mecanización en el proceso de la producción agropecuaria constituye una vía fundamental para el desarrollo ulterior de la agricultura y la satisfacción de las demandas de productos agropecuarios. La intensificación de la producción agrícola, pretendiendo una productividad eficiente y sustentable por cada hectárea del suelo en explotación, es concomitante con la mecanización y con la implementación de una tecnología progresiva 
de máquinas en el cultivo de plantas y en la ganadería (Jróbostov, 1989). Tomando como base el estudio minucioso de los índices técnicos y económicos del trabajo de los conjuntos de los grupos de máquinas para cultivar las plantas del cumplimiento de procesos separados y los índices de aprovechamiento de todo el parque de máquinas durante el año y por periodos, se pone de relieve la experiencia positiva en la organización de la producción, se descubren las reservas y se indican los caminos a seguir para su empleo (Jróbostov, 1989). Hay que recordar que el tractor y sus implementos tienen que renovarse, ya que su periodo determinado de vida útil es corto, y debe buscarse incansablemente la manera de alargar al máximo ese lapso sin menoscabo de la calidad, eficiencia y economía del trabajo que realiza. Es aquí donde el estudio de la productividad del conjunto máquina-tractor adquieren capital importancia como instrumento de aplicación práctica para la mejor utilización de los recursos destinados a la agricultura (Soto, 1983). La mecanización agrícola ha tenido efectos de largo alcance en la agricultura, pues facilita una agricultura eficiente y más productiva en las fincas mexicanas permitiendo que la población quede con más tiempo disponible para otras ocupaciones. Este cambio ha provocado que nuestro país pase de ser una nación casi totalmente agrícola a una potencia industrial (Soto, 1983). De 1960 a 1970 la agricultura mundial tuvo una incorporación de 15000000 de tractores y en 1982 de más de 26000000 derivándose de los datos obtenidos que por cada 100 ha de suelo cultivado se tenían 2.1 tractores. Sin embargo, resultados más irregulares en estos índices los presentan regiones del tercer mundo; por ejemplo, África tiene 0.6 tractores/100 ha; América Latina 0.57 tractores/100 ha, Asia 0.11 tractores/100 ha y países desarrollados capitalistas como la República Federal de Alemania con 16 tractores/100 ha; este país tiene una potencia media en sus tractores de $24.2 \mathrm{~kW}(33.0 \mathrm{hp})$, que aumenta la densidad de tractores por cada 100 ha (Gutiérrez, 1990). Según datos aportados por Negrete (2006), en México durante 1982 se tenían en activo 157967 tractores con una potencia de $4926470 \mathrm{~kW}$ (6700 $000 \mathrm{hp}$ ) con un promedio de $30.8 \mathrm{~kW}$ (42 hp) por tractor en 1988. El programa de desarrollo rural integral estimó para ese año una necesidad de 19729 tractores de los que 14572 eran para reposición y 5157 para incrementar el parque. Según el Censo Nacional Agropecuario VIII (2003) se tenían en el país 177000 tractores; de este total 25000 estaban fuera de servicio por deterioro. En 1995 se estimó un total de 190200 tractores a nivel nacional y para 2011 en México existía un parque de tractores de 238 830, cuyo valor promedio de adquisición era de MXN 375000 a la fecha, 54\% ya rebasó su vida útil (Sagarpa, 2010a), citado por Ayala Garay et al. (2011).

El mayor pico de tractores en la República Mexicana se tuvo en 1991 con un total de 317313 tractores en servicio y a partir de ese año ha ido en decremento el parque activo. También es bueno resaltar que desde 1997 el mercado mexicano (Palacios et al., 2003; Palacios Rangel et al. 2012), es muy estable y reporta ventas promedio de 10000 a 11000 tractores anualmente. De acuerdo con Flores et al. (2007), citado por Ayala Garay et al. (2011), las principales marcas de tractores que consume el mercado mexicano son John Deere, New Holland, International Harvester, Massey Ferguson. Hasta 2008, la tasa de crecimiento tanto de importaciones como de exportaciones ha sido para este último de $13.33 \%$ y para el primero de un 4.32\%, según Ayala Garay et al. (2011). En este aspecto, se puede plantear que los agricultores mexicanos utilizan tractores, cuya potencia fluctúa entre 44 y 62.5 kW (60-85 hp), según Flores et al. (2009). Sagarpa (2011) reporta que en 2010 las casas vendedoras de tractores agrícolas se reparten las ventas de la siguiente manera: New Holland 26\%, Massey Ferguson 27\%, John Deere 38\%, y 9\% restante entre las demás expendedoras de tractores y máquinas agrícolas. Como dato adicional, en 2008 México importó alrededor de 28000 tractores y exportó aproximadamente 21 000 unidades. De acuerdo con Negrete (2006), existen algunos índices que son importantes cuando se quiere conocer el nivel de mecanización de algunos países, y en particular de nuestro país. Por ejemplo, México tenia 84 ha/tractor en 2008, mientras que Europa 28 ha/tractor; Estados Unidos de América 37 ha/tractor y América Latina $95 \mathrm{ha} /$ tractor. Otro índice importante es la potencia por hectárea; al respecto, es necesario resaltar que los valores de los países industrializados están en alrededor de $0.73 \mathrm{~kW} / \mathrm{ha}$ ( $1.0 \mathrm{hp} / \mathrm{ha})$. México, de acuerdo con cifras de Reina Castro y Hetz (2012), tiene $1.04 \mathrm{hp} / \mathrm{ha}(0.77 \mathrm{~kW} / \mathrm{ha})$ y Venezuela $1.06 \mathrm{hp} / \mathrm{ha}(0.79 \mathrm{~kW} /$ ha), lo cual contrasta con países (índices más bajos) de América Latina: Bolivia con $0.10 \mathrm{hp} / \mathrm{ha}(0.08 \mathrm{~kW} / \mathrm{ha})$ 
y Perú con $0.19 \mathrm{hp} / \mathrm{ha}(0.14 \mathrm{~kW} / \mathrm{ha})$. Los valores que presenta México son de un nivel bastante aceptable de mecanización agrícola.

Para 2004, América Latina y el Caribe tenían 95 ha/tractor; en este caso, la superficie agrícola que se toma como patrón es la cantidad de suelo cultivado, de acuerdo con datos proporcionados por Negrete et al. (2013), el índice de mecanización de hectárea por tractor agrícola en el centro del país es de $104.3 \mathrm{ha} /$ tractor, el parque de tractores de 79600 unidades y la superficie mecanizable de 8300000 ha. En cuanto a la potencia total, Argentina mostró, según lo refiere Reina Castro Hetz (2012), 20949000 kW México 21117850 kW y Venezuela $2695000 \mathrm{~kW}$. Se debe plantear que las labores agrícolas no se pueden distribuir en el tiempo, ni a voluntad, ni uniformemente, sino que es necesario realizarlas en lapsos determinados dependientes de la estación del año, de las condiciones climatologías y del suelo, factores que en la mayoría de los casos no pueden ser controlados plenamente por el hombre y que incidirán sobre el proceso técnico-económico de la explotación del parque de máquinas agrícolas (Gutiérrez, 1990). La mecanización no es una actividad que se produce en forma aislada, puesto que existe una estrecha relación entre el proceso como tal y las características de los diferentes cultivos debido a que no presentan iguales requisitos, así como distintas variedades de plantas dentro de las mismas especies y tampoco manifiestan el mismo comportamiento de una u otra forma sobre la mecanización (Gutiérrez, 1990).

Con base en lo anterior, el objetivo del artículo es conocer el nivel de mecanización agrícola que posee el municipio de Zinacantepec, Estado de México, así como establecer un comparativo a nivel regional y mundial de los países que poseen mayor índice de mecanización.

Para elaborar este artículo, se analiza la situación actual y perspectiva de la mecanización agrícola en México, se utiliza una metodología basada en una encuesta y se presentan los resultados obtenidos, así como un apartado de conclusiones y prospectiva.

\section{Situación actual y perspectiva de la mecanización agrícola en MéXico}

El sector agropecuario mexicano ha enfrentado transformaciones profundas durante las tres últimas décadas; el continuo proceso de urbanización, el intenso proceso de globalización y las transformaciones demográficas han configurado un nuevo entorno para el sector agropecuario (Escalante y Rello, 2000), el cual se caracteriza por cambios tecnológicos que redundan en mejoras de la productividad, nuevos cultivos que se ajustan a las exigencias de un mercado internacional, modificaciones genéticas que mejoran las variedades de los productos, esquemas organizacionales que dinamicen las formas de comercialización y modifiquen los métodos de inserción en el mercado mundial e incluso el surgimiento esquemas de desarrollo rural modernos (Escalante y Rello, 2000; Ibarra y Acosta, 2003; Moreno et al., 2004). Ocampo y Palacios (2003) señalan que en México existen aproximadamente 21900000 ha agrícolas, de las cuales se estima, considerando las características de la pendiente, que 18600000 ha son potencialmente mecanizables (Moreno et al., 2004). Actualmente, hay 223526 tractores en servicio en México (Negrete, 2013), pero 54\% ya rebasó su vida útil, según lo reporta Ayala Garay et al. (2011). Dentro de los problemas que enfrentan los productores del campo mexicano, se encuentra la falta de liquidez para la compra de maquinaria agrícola (el precio promedio mínimo por tractor varía desde MXN 375000 a MXN 800 000); además de la inversión, el incremento en los costos de combustible y operación de los tractores resulta costosa, por lo que se convierten en limitantes para la adquisición y mantenimiento de los equipos (Ayala Garay et al., 2011).

Por otro lado, las importaciones se han incrementado, ya que éstas crecieron a una tasa media anual de $4.32 \%$ entre 1980 y 2008 y en promedio se importaron 25000 tractores en el periodo 2006-2008 (Ayala Garay et al., 2011).

$\mathrm{Al}$ respecto, se puede plantear que el crecimiento del parque de máquinas y tractores agrícolas en el agromexicano necesita tener mecanismos apropiados que le den certeza, que los tractores y máquinas agrícolas que estén adquiriendo sean de óptima calidad y que cumplan con las especificaciones mínimas esenciales de explotación y fiabilidad mecánica durante su vida útil. 


\section{Materiales y Métodos}

Esta investigación se realizó en el municipio de Zinacantepec, Estado de México, ubicado en latitud norte; los meridianos $99^{\circ} 42^{\prime}$ y $99^{\circ} 55^{\prime}$ de longitud oeste y altitud entre 2600 y $4300 \mathrm{msnm}$. En el territorio municipal predomina el clima semifrío subhúmedo, con temperaturas en el verano de $28^{\circ} \mathrm{C}$ y en invierno hasta $-5^{\circ} \mathrm{C}$. Las presencias de los vientos van de oeste a este y viceversa; los meses de diciembre, enero, febrero, marzo y abril tienen la estación más seca. Existe una precipitación anual de 800 a 1500 mm. Las precipitaciones se presentan en los meses de mayo a octubre. Zinacantepec posee 44 localidades y una población total de 188927 habitantes. En cuanto a su territorio, cuenta con 16212 hectáreas destinadas al uso agropecuario, 32.25\% está destinado al cultivo de temporal y $9.94 \%$ de cultivo de riego, clasificadas en agricultura manual estacional con un $41.38 \%$ (agricultura mecanizada continua 25.04\%, agricultura mecanizada estacional 15.34\%, agricultura de tracción animal estacional 6.78\% y no apta para la agricultura 11.46 (Plan de Desarrollo Municipal Zinacantepec 20162018). La actividad agrícola mecanizada corresponde a un $40.38 \%$; esto implica que, al indagar los índices técnicos-económicos de mecanización, se puedan elaborar propuestas que conlleven al uso eficiente de los tractores y máquinas agrícolas en la región.

La metodología se basó fundamentalmente en la realización de una encuesta aplicada a productores agropecuarios, dueños de tractores y máquinas agrícolas; para ello, se diseñó un instrumento (cuestionario) como herramienta principal de trabajo. Antes de comenzar la encuesta realizada, se consultó con la Sedagro (Secretaría de Desarrollo Agropecuario del Gobierno del Estado de México), Sagarpa, el H. Ayuntamiento del Municipio de Zinacantepec, Estado de México, y las principales agencias distribuidoras de máquinas agrícolas del municipio de Toluca, Estado de México (New Holland, John Deere y Massey Ferguson) con la finalidad de analizar las bases de datos de máquinas agrícolas en posesión de productores agropecuarios, ex profeso del municipio de Zinacantepec, con un censo en el periodo 1999-2015 de 304 tractores. El tamaño de la muestra se calculó en función de una unidad mínima y las comunidades elegidas se tomaron al azar, con un nivel de confianza de $95 \%$ y como límite del error muestral se tomó 9\%. Para obtenerse el tamaño de la muestra, se utilizó la fórmula siguiente, la cual es utilizada para poblaciones finitas y se conoce el total de unidades de observación que la integran:

$$
n=\frac{N \sigma^{2} Z^{2}}{(N-1) e^{2}+\sigma^{2} Z^{2}}
$$

Dónde: $n$ tamaño de la muestra, $N$ tamaño de la población, desviación estándar de la población que, generalmente cuando no se tiene su valor, suele utilizarse un valor constante de $0.5, Z$ valor obtenido mediante niveles de confianza. Es un valor constante que si no se tiene su valor, se lo toma en relación al $95 \%$ de confianza equivale a 1.96 (como más usual) o en relación al 99\% de confianza equivale 2.58, valor que queda a criterio del investigador, $e$ límite aceptable de error muestral que, generalmente cuando no se tiene su valor, suele utilizarse un valor que varía entre $1 \%$ (0.01) y (0.09), valor que queda a criterio del encuestador (Aguilar-Barojas, 2005).

Los propietarios de tractores agrícolas a muestrear se seleccionaron con el empleo de un método aleatorio simplificado y el padrón de propietarios con tractores fueron solicitados en la ventanilla de información de Sedagro. Estos mismos datos de los últimos diez años se solicitaron en las empresas de venta de tractores agrícolas de la ciudad de Toluca de Lerdo, en febrero de 2017; al realizar el cálculo de la muestra, el tamaño óptimo de la misma fue de $85 \pm 2$ tractores y con base en estos valores se realizó la encuesta.

La caracterización del nivel de mecanización para el municipio de Zinacantepec se elaboró en función de la cantidad de tractores agrícolas y sus diferentes potencias, número de implementos para cada uno de ellos, 
índice de equipamiento energético, moto-horas trabajadas al año, versión de los sistemas de rodajes de los tractores, potencia por hectárea de suelo cultivado, promedio de moto-horas trabajadas por tractor, promedio de años por tractor.

\section{Resultados Y Discusión}

Con base en la encuesta realizada a 84 productores se contabilizaron 85 tractores, lo cual implica que uno de los productores tiene más de un tractor. En la gráfica 1 se muestra la cantidad de implementos agrícolas por categorías que se observaron en las encuestas aplicadas, lo cual arroja un total de 388 implementos: 82 arados en una relación con los tractores de 0.96 arados por tractor. En las labores de preparación complementaria del suelo se tienen 83 rastras lo que significa que la relación rastra-tractor es de 0.99 , lo que muestra que prácticamente cada tractor posee una rastra. En referencia a las sembradoras, sólo $85 \%$ de los tractores tienen una sembradora, pues existen 73 , lo cual da una relación sembradora tractor de 0.85 sembradora por tractor si se tiene en cuenta que ésta es una de las máquinas agrícolas más costosas y de más difícil manejo y mantenimiento. En esta zona agrícola se tienen 28 fertilizadoras, siendo la relación con respecto al tractor de 0.33, lo cual es baja, teniendo en cuenta que el cultivo de maíz, predominante en esta zona agrícola, necesita que se le apliquen fertilizaciones después de los 40 hasta los 60 días de su emergencia, dado el tiempo en función de la cantidad de humedad que tenga el suelo, ya que es una práctica extendida entre los agricultores de la región y otra parte de la fertilización la aplican en la segunda escarda, pero en la generalidad de los casos no puede ser mecanizada por la altura de la planta y la no existencia de tractores de alto porte, de acuerdo con la experiencia de los autores. En la actualidad se están adoptando nuevas tecnologías de preparación primaria de suelos en el Valle de Toluca y experimentos realizados en la Facultad de Ciencias Agrícolas de la Universidad Autónoma del Estado de México, y trabajos de extensionismo con los agricultores se han comenzado a utilizar multiarado-subsolador y la relación es de 0.30 multi-subsolador/tractor (Vaca García et al., 2014); se debe plantear que todavía existe resistencia en la adopción a las nuevas tecnologías de corte vertical y no inversión del prisma de suelo, así al menor tiempo en la preparación de suelos y ahorro de combustible (Gutiérrez, 2004).

En la gráfica 1 se muestran las cantidades de implementos agrícolas y el porcentaje de estos respecto al total que suman la cantidad de 388, y de esto se plantea la relación implementos agrícolas por tractor, la cual exhibe 4.56 implementos por tractor; por debajo de lo planteado por Garrido (1984) e Iofinov (1984), la relación debe ser de seis implementos por tractor para que, de esta manera, tenga una plena capacidad de trabajo la mayor parte de los ciclos agrícolas y como es lógico esta relación estará en dependencia del tipo de cultivo. Por otra parte, es importante la cantidad de suelo cultivado por tractor agrícola, la cual presenta la cifra de $13.08 \mathrm{ha} /$ tractor; en relación con esto, Ramírez (2007) plantea que debido a la estructura agraria en México prácticamente se hace inviable la mecanización agrícola, ya que impera el minifundio. Y, remitidos a los resultados, se observa que el municipio de Zinacantepec tiene condiciones similares a lo que plantea este investigador, puesto que la mayor parte de los productores que poseen tractores, sus superficies de suelo están comprendidas entre 2 y 9 ha y más adelante se explica la tenencia de la tierra. En este tenor, según la FAO (2011), citado por Larqué Saavedra (2012), menciona que para que un tractor agrícola sea rentable debe de tenerse una relación superficie/tractor de aproximadamente $50 \mathrm{ha} /$ tractor como promedio, teniendo en cuenta la propiedad ejidal, rural y comunal. En la investigación realizada por Larqué Saavedra (2012), citando a Negrete (2006), indica que a partir de una frontera agrícola con 24000000 ha, con una superficie mecanizable de 18600000 ha, se requeriría del orden de 360000 tractores con potencia de 36.8 a 44 kW (50 a 60 hp), lo que significa un índice de mecanización de aproximadamente de 51 ha/tractor, lo cual corrobora la subutilización de los tractores agrícolas en la zona de estudio. Según Masera (1990), para hacer rentable un tractor mediano $44 \mathrm{~kW}(\approx 60 \mathrm{hp})$, es necesario tener una superficie de cultivo de 25 ha, hecho que es ratificado por Lara López (2000), quien en un estudio realizado 
encontró que el punto de equilibrio para un tractor de categoría mediana armado en México dedicado a la maquila de las labores agrícolas es de 31 ha.

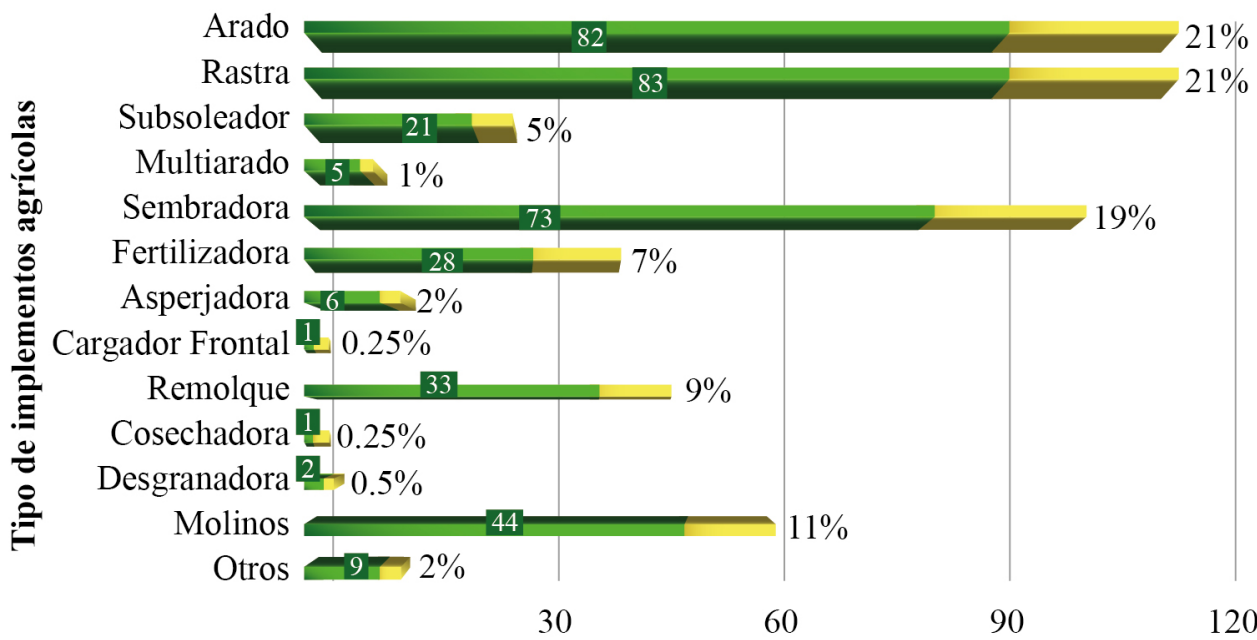

Número de implementos agrícolas $\square$ Porciento

\section{GRÁFICA 1}

Número de implementos agrícolas

Fuente: elaboración propia.

Como se observa en la gráfica 2, la posesión de la tierra en este municipio se distribuye principalmente de la siguiente manera: $2.38 \%$ posee 50 o más hectáreas; $4.76 \%$ con 28.0 ha y 13.0 ha; $26.19 \%$ con 9.0 ha; $27.38 \%$ con 6.0 ha, $21.42 \%$ con 3.0 ha y $3.57 \%$ con 1.0 ha.

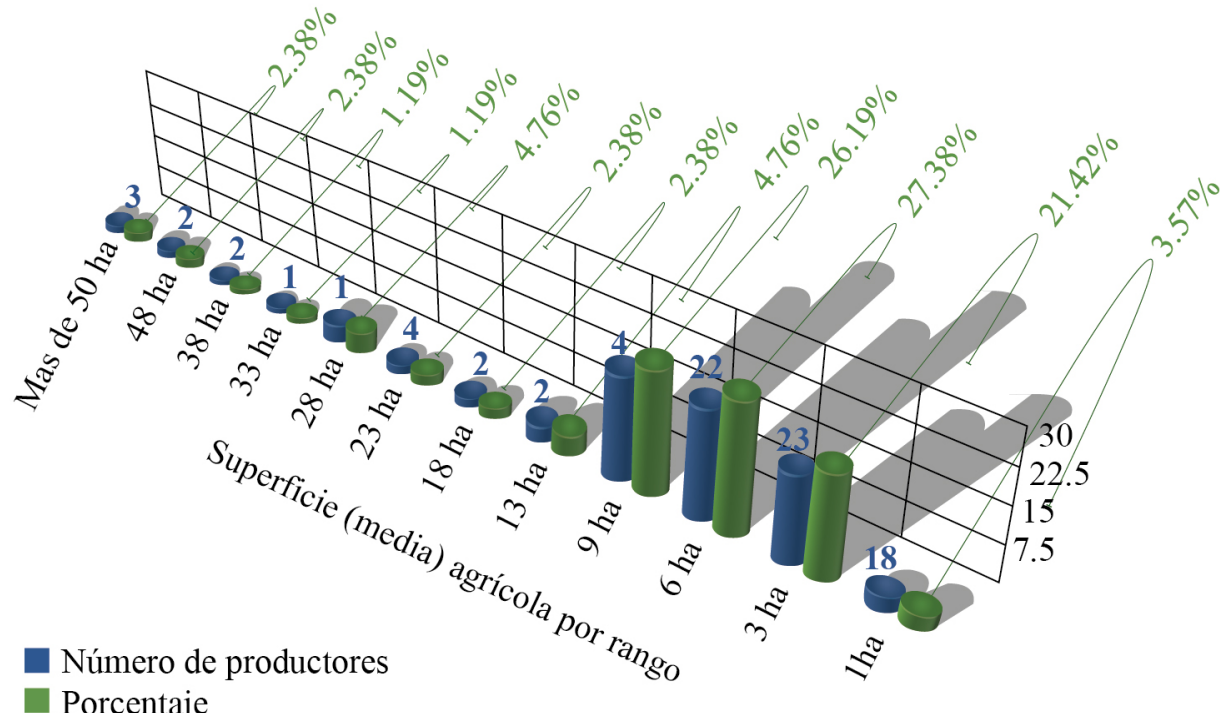

GRÁFICA 2

Distribución-porcentaje de productores agrícolas en relación con la superficie Fuente: elaboración propia. 
Los productores en referencia (gráfica 3) configuran la tenencia de la tierra en la siguiente proporción: ejidal 90.48\%; comunal $5.95 \%$ y propiedad rural $3.57 \%$ y cada uno de ellos tienen tractores. Estos datos no indican la subutilización que se está haciendo de la maquinaria que poseen y está en contra de lo planteado por Lara (2000) y Masera (1990), referidos anteriormente. Es importante señalar que en el rango de 1.0 a 13.0 ha, la usufructúan los propietarios de 66 tractores, es decir, se encuentran concentrados en ejidatarios, lo cual refuerza la investigación realizada por Lara López (2000).

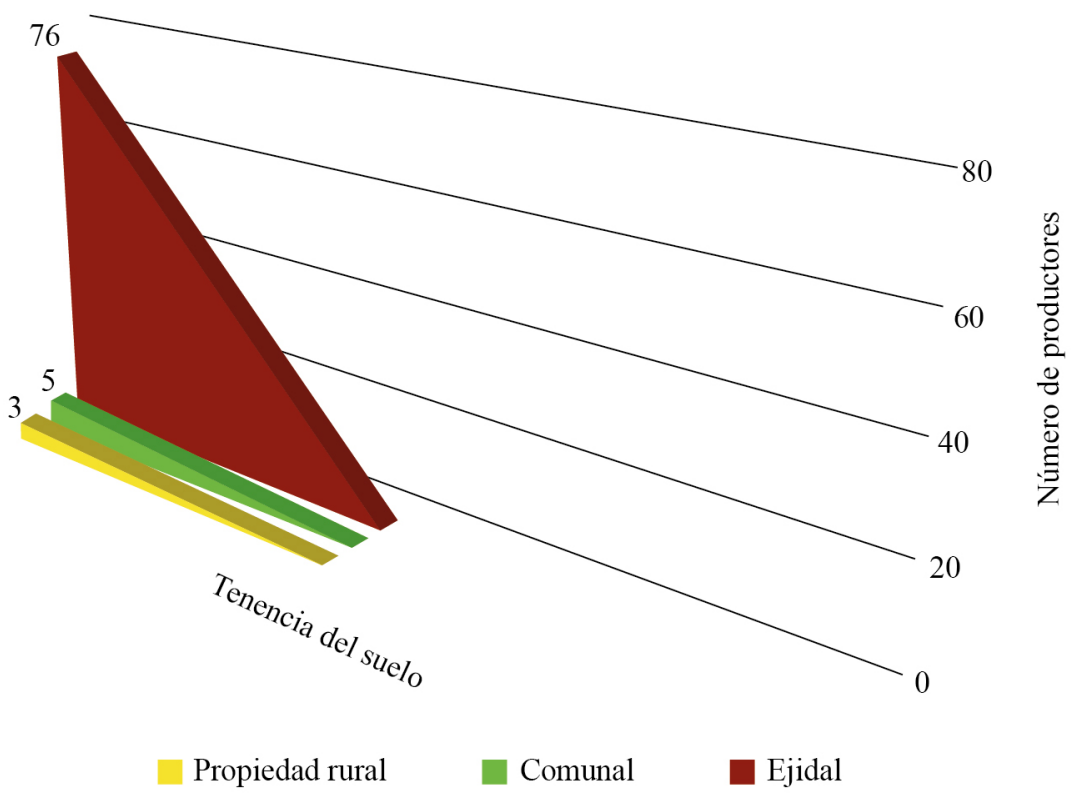

GRÁFICA 3

Número de productores por tipo de tenencia de la tierra

Fuente: elaboración propia.

La gráfica 4 indica la cantidad de tractores y el porcentaje que significan para las diferentes potencias en hp y, como se puede observar, en la gráfica representativa, en primer lugar, se tiene un tractor con $95.6 \mathrm{~kW}$ ( $130 \mathrm{hp}$ ), lo cual significa un $1.17 \%$ del total de tractores. En el número 2 de la escala horizontal, se tienen un tractor con 82.2 $\mathrm{kW}(120 \mathrm{hp}$ ) y esto representa un 1.17\% de los tractores con esta potencia, 13 tractores que significan el 15.29\%, están ubicados en la potencia de $88.2 \mathrm{~kW}(110 \mathrm{hp})$ y en el "pico" máximo de todos estos tractores, se ubican en los 90 hp con un número de 29 tractores; esto significa un $34.11 \%$, les siguen en potencia los tractores de $80 \mathrm{hp}$ con 24 tractores que significa un 28.23\%, así como 13 y 4 tractores con potencias respectivas de 51.4 y $44.1 \mathrm{~kW}$ (70 y $60 \mathrm{hp}$ ) y con porcentajes correspondientes de 15,29 y $4.71 \%$ respectivamente.

En la gráfica 5 se observa que los mayores volúmenes de tractores se concentran en la potencia correspondiente a $66 \mathrm{~kW}$ (90 hp) coincidente con la investigación realizada por Larqué Saavedra et al. (2012), con potencias de tractores de 51.4 a 80.8 ( 70 a 110 hp) y estos tienen un $77.63 \%$; esta información corrobora a la investigación llevada a cabo en el municipio de Atlacomulco, Estado de México, donde se arrojan datos de 78.96\%; no muy distinto de estos resultados en el municipio de Zinacantepec. El patrón que se tiene en estos lugares prácticamente es el mismo y es válido para ambos lugares, lo planteado en los análisis que realiza esta investigadora. Teniendo en cuenta la suma de las potencias del conjunto de tractores, se tiene un total de $5272 \mathrm{~kW}(7170 \mathrm{hp})$ y como promedio se tienen $5.29 \mathrm{~kW} / \mathrm{ha}(7.20 \mathrm{hp} / \mathrm{ha})$, muy por encima de la media nacional, que según la Faostat (División de Estadística Organización de las Naciones Unidas para la Alimentación y la Agricultura, 2017), es de $1.04 \mathrm{hp} /$ ha y algo más elevado de los encontrado por SánchezHernández (2014) en los municipios de Texcoco y Amecameca, el cual fue de 3.38 y $4.7 \mathrm{~kW} / \mathrm{ha}(4.6$ y 6.4 
hp/ha), en esta investigación Sánchez-Hernández (2014), plantea que de acuerdo a lo encontrado por Gaytán Ruelas (2007) la potencia por hectárea recomendada está en $0.73 \mathrm{~kW} / \mathrm{ha}(1.0 \mathrm{hp} / \mathrm{ha})$. La potencia como promedio por tractor en el municipio de Zinacantepec está en $62.35 \mathrm{~kW}(85.35 \mathrm{hp})$, lo cual se acerca bastante a lo identificado por Reina Castro (2004) y Reina Castro et al. (2012), ya que en su investigación arrojó un promedio de $70 \mathrm{~kW}(87.0 \mathrm{hp})$ en México.

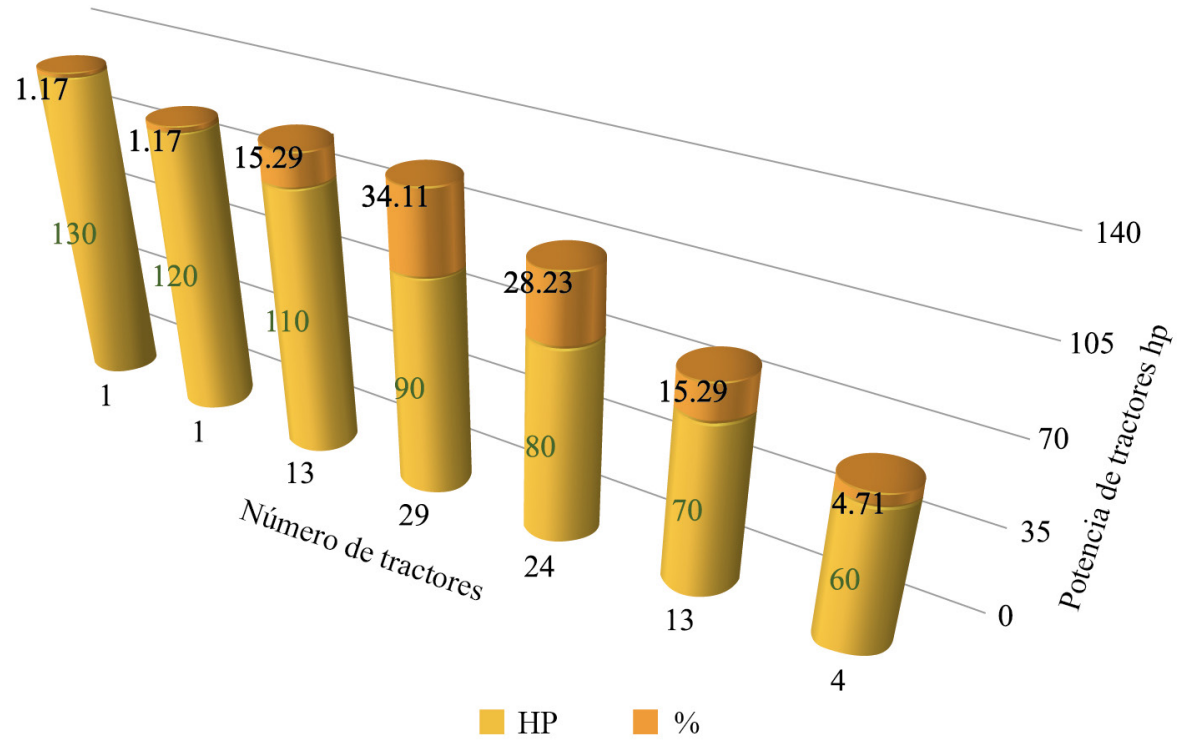

GRÁFICA 4

Relación de tractores-potencia $\mathrm{kW}(\mathrm{hp})[\mathrm{un} \mathrm{kW} \approx 1.36 \mathrm{hp}]$

Fuente: elaboración propia.

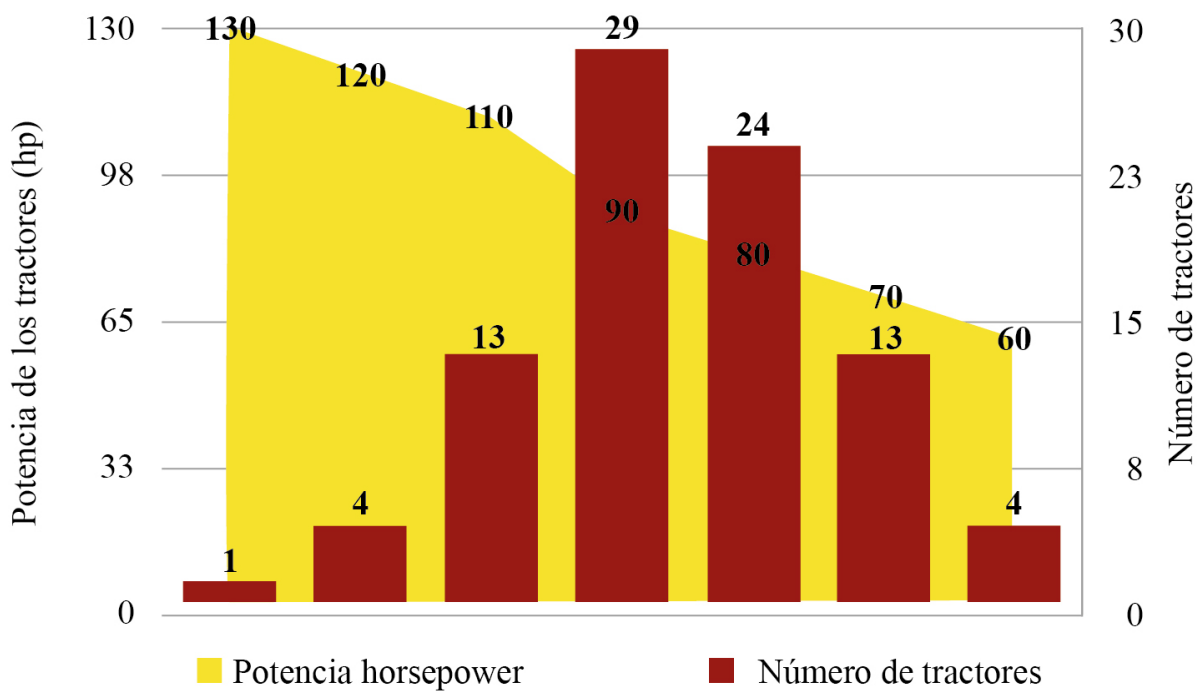

GRÁFICA 5

Concentración tractores-potencia

Fuente: elaboración propia.

En la gráfica 6 se puede observar la cantidad de moto-horas que se han trabajado por los tractores durante su vida activa en las labores agrícolas sin tener en cuenta el envejecimiento de los tractores, los 
cuales promedian 3200 moto-horas en 7.73 años de explotación. De acuerdo con el cálculo anterior, los tractores trabajan anualmente un promedio de 414 moto-horas en el municipio de Zinacantepec y en España los tractores similares trabajan anualmente un promedio de 512.9 moto-horas, tractores que tienen una potencia promedio de 70 a $89 \mathrm{hp}$, que en lo fundamental son los identificados en la actual investigación (Ministerio de Agricultura, Pesca y Alimentación de España, 2018), en una investigación realizada por Negrete (2013), cita varios investigadores, en donde Jasmen (2004) plantea que los tractores deben trabajar 500 moto-horas anuales y por otro lado Slater (2008) plantea $836 \mathrm{~m}-\mathrm{h}$ (moto-horas); sin embargo, Cadena (1997) y Lara (2000) plantean un uso anual de $1000 \mathrm{~m}$-h, de esto se deriva que existen disímiles criterios, pero de todas maneras los tractores producto de esta investigación trabajan menos de los planteado por estos investigadores.

Total moto-horas trabajadas por grupo de tractores

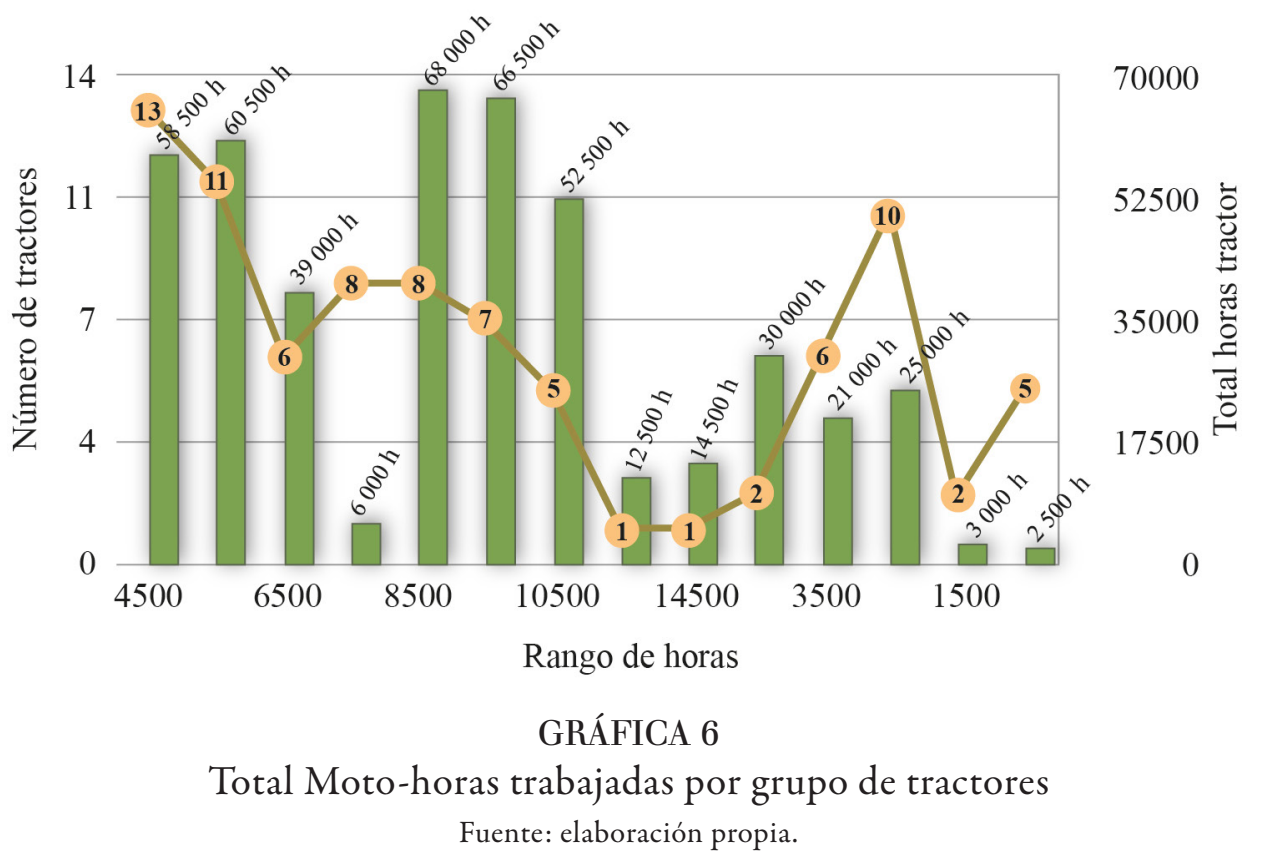

En la gráfica 7 se muestran la cantidad de tractores y el año en el cual se comenzaron a utilizar, con la excepción de los tractores que se compraron en 2005 o antes, el cual se eleva a la cifra de 27 tractores y que, de acuerdo con el cálculo realizado, los tractores tienen un promedio de 7.73 años, como fue señalado. Según Selivanov (1972), un tractor debe durar aproximadamente 12 años de utilización a plena capacidad y después de este periodo debe existir una base de producción que sería capaz de fabricar en este tiempo o plazo por lo menos un mismo tractor nuevo en lugar del desgastado. En este aspecto, se plantea que el costo-beneficio que proporcionan los tractores, un $20 \%$ debe estar dirigido a la renovación, un $44 \%$ para las piezas de repuestos y un $36 \%$ para gastos de reparación y mantenimiento técnico. Por otro lado, se puede plantear que para algunas máquinas agrícolas es propio un alto peso específico de volúmenes de trabajo para el mantenimiento técnico y las reparaciones (hasta el $50 \%$ con un plazo de servicio de 8 a 10 años), ya que durante su utilización se requiere efectuar frecuentemente tales operaciones. Un empleo racional y de mantenimiento preventivo y correctivo en los tractores y máquinas agrícolas asegura el aumento más racional de sus plazos de servicio, la disminución del ritmo general de desgaste físico y moral de los tractores y las máquinas agrícolas.

Cuando se identificaron los tractores por las diferentes versiones en sus sistemas de rodaje (gráfica 8), se observó que 


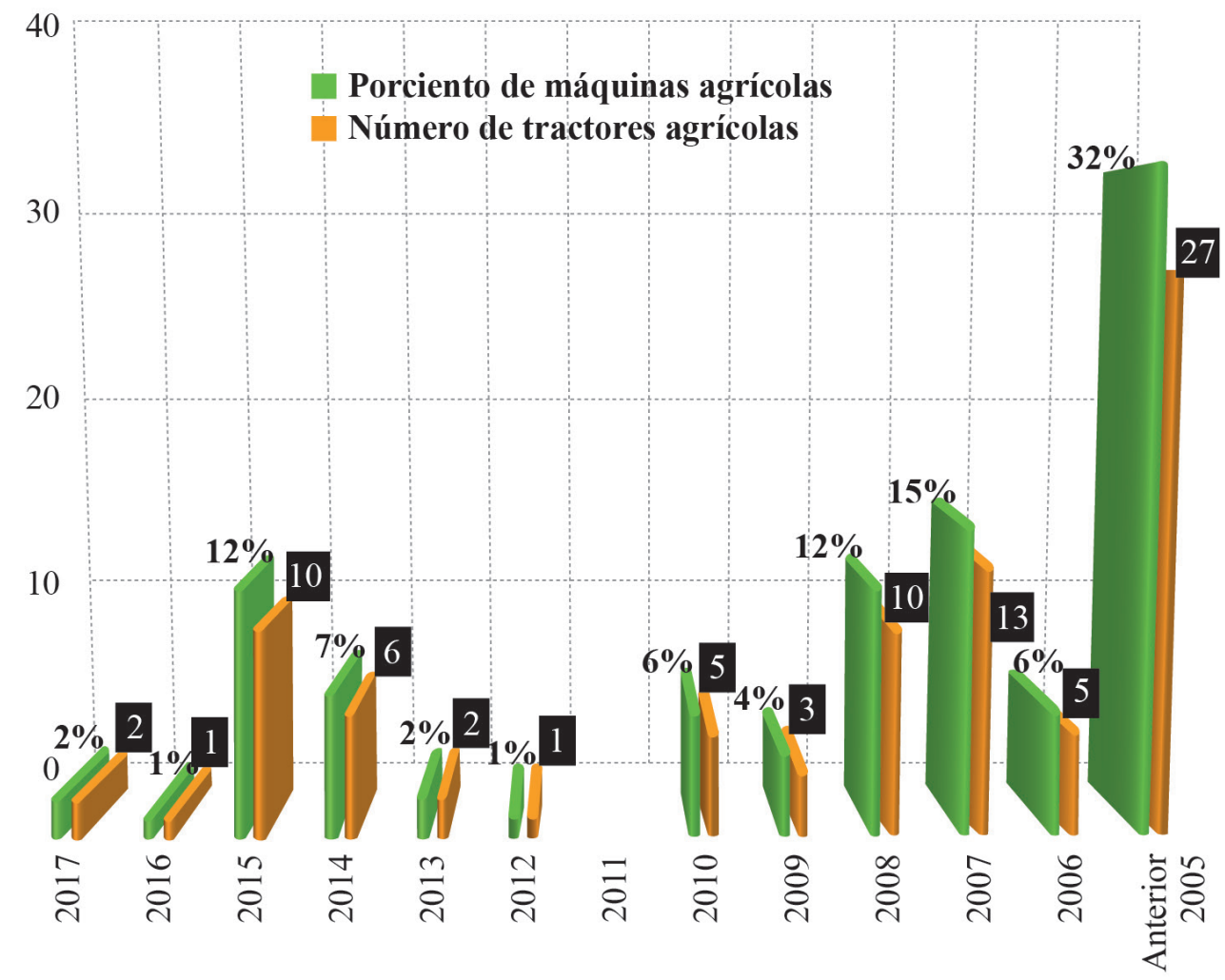

Fecha de adquisición-operación de la máquina agrícola

GRÁFICA 7

Moto-horas trabajadas por grupo de tractores

Fuente: elaboración propia.

$52 \%$ de los tractores tienen fórmula $4 \times 2$. Esta versión es la más barata y hasta se pudiera plantear que son los más versátiles y universales, ya que con ellos se pueden realizar toda una serie de trabajos agrícolas, además de que sus mantenimientos son de menor costo; sin embargo, tienen una menor tracción y con altas sobrecargas teniendo la posibilidad de vencer con menor facilidad las resistencias externas que les ofrecen los implementos y máquinas agrícolas que realizan la tarea agrícola y sobre esto se incrementa el resbalamiento o patinaje, lo cual ocasiona un mayor desgaste en los neumáticos motrices y por ende una mayor compactación al suelo agrícola. El segundo lugar lo ocupan los tractores con fórmula $4 \times 4$, los cuales a veces no pueden cumplir a cabalidad todas las tareas que se tienen en el campo de cultivo; no obstante, por tener una mayor área de contacto con el suelo, lo compactan menos, tienen una mayor tracción, pues tienen menor resbalamiento. Los tractores con esta versión en su sistema de rodaje pueden realizar con mayor rapidez las labores donde sea necesario tener una mayor tracción y por lo tanto esto ayuda a un menor consumo de combustible, aunque su mantenimiento técnico es algo más costoso. Y la otra versión o fórmula de su sistema de rodaje es la de $3 \times 2$, en la cual sólo existe un tractor (1.0\%), muy utilizada en tareas especiales para cultivos de alto porte.

La gráfica 9 nos permite analizar y describir lo siguiente: los costos en el manejo, explotación, mantenimiento y reparación resultan aún más costosos por no realizar estrategias de capacitación para la operación eficiente de los tractores agrícolas, así como no conocer las normas en cuanto a un mantenimiento preventivo; en relación con la capacitación del propietario, indica 57\% no fue capacitado y 43\% de propietarios sí recibió capacitación para la operación del tractor. En cuanto al conocimiento de las normas de mantenimiento preventivo por parte de los propietarios, 69\% indica que no conoce dichas normas, sólo un 30\% de los productores respondió que sí las conocen; es decir, este porcentaje manifiesta una cultura de mantenimiento preventivo y de ellos 50 propietarios acuden a las agencias autorizadas para la reparación de desperfectos mecánicos. 


\section{Rodaje}

\section{Otros}

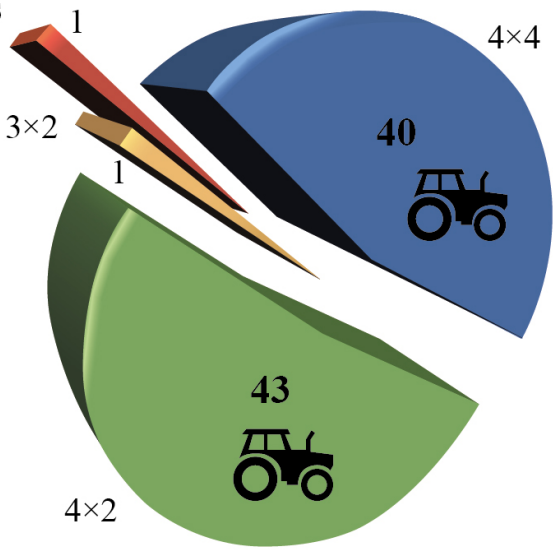

40 Tractores $4 \times 4$

43 Tractores $4 \times 2$

1 Tractor $3 \times 3$

1 Tractor de otro tipo de rodaje

GRÁFICA 8

Relación de tractores-rodaje

Fuente: elaboración propia.

Capacitación

Conoce las normas de mantenimiento preventivo

Realiza el mantenimiento preventivo

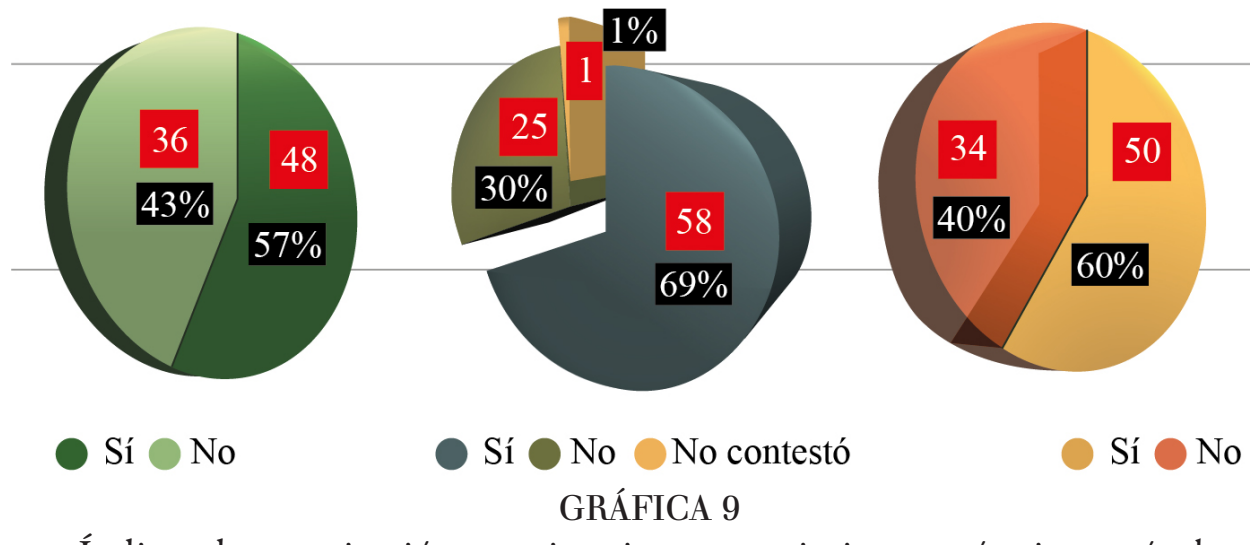

Índices de capacitación-propietario, mantenimiento-máquina agrícola

Fuente: elaboración propia.

\section{Análisis prospectivo}

El reto futuro del uso de la mecanización agrícola en el mundo, y en particular en México, se basa fundamentalmente en el binomio productor-máquinas agrícolas eficientes (uso de la robótica) y sustentables, por lo que el gobierno federal debe generar políticas públicas agrícolas ad hoc al nivel socio cultural del agricultor (desarrollo rural sostenible) en relación con los tipos de suelos agrícolas de la región. El encarecimiento del tractor, máquina e implementos agrícolas o el crédito agrícola no adecuado en estímulos de tipo agrotécnico, Fiscal y cero subsidios al combustible para el agricultor han propiciado que la maquinaria agrícola se "envejezca" y por consiguiente no se modernice el parque de maquinaria. Es notorio que el agricultor día a día deja de invertir en el campo agrícola y en sus procesos de mejora que abarcan la preparación de suelos, administración, uso de maquinaria agrícola eficiente, apertura a la vinculación con centros de investigación agrícola, fábricas y concesionarias de tractores, equipos 
para modernizarse y también dar certidumbre al valor agregado de sus cosechas. Otro fenómeno a estudiar y que va de forma concomitante a la productividad agrícola es estimular la mano de obra en particular a los operadores. En cuanto a la investigación realizada, ésta debe ser coadyuvante con estudios similares, por lo que se propone la integración del "Polo Regional Agrícola del Valle de Toluca", que comprende a los municipios de Almoloya de Juárez, Toluca, Metepec, Lerma, San Mateo Atenco, Zinacantepec, Rayón, Calimaya, Tenango del Valle, entre otros, y que en su conjunto se determine el tipo de máquinas agrícolas que requieren los productores agropecuarios.

\section{Conclusiones}

Los implementos de preparación primaria y complementaria de suelos (arado y rastra) muestran las relaciones más altas (implemento/tractor) de 0.97 y 0.98 respectivamente, seguida de las sembradoras con un índice bastante bajo de 0.86. La relación general implemento/tractor es de 4.56, muy por debajo del óptimo que debe ser 6:1. Estas relaciones nos ayudan a concluir que las labores principales en las cuales están ocupadas las máquinas agrícolas y los tractores son las mencionadas. Se ha encontrado que en la región encuestada existe una relación de 13.08 ha/tractor y, según los criterios de la FAO (2017), esta relación es pequeña y por lo tanto el binomio tractor-implemento es subutilizado. El promedio de edad que tienen los tractores es de 7.73 años de utilización y la cantidad de motohoras promedio del tractor es de aproximadamente 414 por año de utilización, cifra por debajo de lo utilizado en un $19.7 \%$ en relación con España que muestra un indicador de 512.9 moto-horas y casi en un $50 \%$ de lo utilizado anualmente en la República Federativa Democrática de Rusia con 950 moto-horas, según criterios establecidos. Otro criterio a tomar en cuenta es que $77.38 \%$ de los tractores se concentra en superficies que fluctúan de 0 a 10 ha y con una potencia promedio de $66 \mathrm{~kW}$ ( $90 \mathrm{hp}$ ). Se desprende de la investigación que un alto porcentaje de agricultores no posee los conocimientos para realizar los mantenimientos técnicos preventivos y correctivos, lo cual conlleva al deterioro prematuro de la fuente de energía del tractor (motor), así como de otros mecanismos fundamentales para una explotación eficiente de las máquinas agrícolas.

\section{Agradecimientos}

Se agradecen los comentarios de los árbitros de la revista que mejoraron sustancialmente el contenido del artículo.

\section{ReFERENCIAS}

Aguilar-Barojas, S. (2005). Fórmulas para el cálculo de las muestras en investigaciones de salud. Salud en Tabasco, 11(2), 333-338.

Ayala Garay, A. V., Audelo Benítez, M. A. y Aragón Ramírez, A. (2011). La situación del mercado de tractores en México, perspectivas y retos en la certificación. Textual, 58, 91- 111.

Escalante, S. R. y Rello, F. (2000). El sector agropecuario mexicano: los desafíos del futuro. Comercio Exterior, $50(11)$.

FAO (Organización de las Naciones Unidas para la Agricultura y la Alimentación) (FAO-FAOSTAT). (2017). Consultado el 29 de noviembre de 2017. Disponible en http://www.redagricola.com/mercado-maquinariaagricola-cifras-evolucion/.

Flores, F. y Schwentesius, R. (2009). Mecanización del agro en México. Agro Rev. Ind. Campo, 54(8), 23-34. Disponible en http://3wméxico.com/2000agro/revpdf/agro54.pdf. 
Gaytán Ruelas, J. G. (2007). Administración de maquinaria agrícola. Apuntes de curso. Ingeniería Mecánica Agrícola. Chapingo, México.

Garrido, P. J. (1984). Implementos y máquinas agrícolas y fundamentos para su explotación. Ed. Científico Técnica Habana, Cuba. 398 páginas.

Gutiérrez, R. F. (1990). Explotación del parque de máquinas y tractores. Monterrey: Universidad Autónoma de Nuevo León.

Gutiérrez, R. F, González Huerta, A. Serrato Cuevas, R. Norman Mondragón, T. (2004). Evaluación tecnológicoexplotativa del conjunto multiarado-tractor. Ciencia ergo-sum, 11(2), 171-176.

Ibarra, D. y Acosta, A. (2003). El dilema campesino. Investigación económica. Vol. LXII. Nº.245, 151-220.

Iofinov, S. A. (1984). Explotación del parque de máquinas y tractores. Moscú: Editorial Kolos.

INEGI. Revista del VIII censo Agrícola y Ganadero (en línea). Disponible: https://www.inegi.org.mx/contenidos/ programas/cagf/2007/doc/hicimos_cagyf.pdf. Consultado en mayo 2018.

Jróbostov, S. N. (1989). Explotación del parque de tractores y máquinas, Moscú: MIR.

Lara López, L. A. (2000). Trends and requirements of Mechanization: The case of Mexico, Proceedings of the 1st Latin-American Meeting of the Club of Bologna, Fortaleza, Brazil.

Larqué Saavedra, B. S., Cortes, E. L., Sánchez, H. L. A., Ayala, G. A. V. y Sangerman Jarquín, D. M. (2012). Análisis de la mecanización agrícola en la región de Atlacomulco. Estado de México. Revista Mexicana de Ciencias Agricolas, 3(4).

Masera, C. O. (1990). Crisis y mecanización de la agricultura campesina. El colegio de México.

Ministerio de Agricultura, Pesca y Alimentación de España (2018). Disponible en https://www.mapama.gob. es/ Consultado en mayo 2018.

Moreno, R. D., Hoyos, F. y Jiménez, R. R G. (2004). Situación de la mecanización agrícola en México. Memoria del Seminario Internacional "La Mecanización Agricola y su Política”. CENEMA, SAGARPA, JIICA. México.

Negrete, J. C. (2006). Mecanización agrícola en México, México, D.F. Rev. Iberoam. CTS. 15 p. Disponible en http://www.revistacts.net/files/Negrete_EDITADO.pdf.

Negrete, J. C., Tavares, M. A. L. y Tavares, M. R. L. (2013). Parque de tractores agrícolas en México: estimación y proyección de la demanda. Revista Ciencias Técnicas Agropecuarias, 22(3).

Ocampo, L. G. J. y Palacios I. (2003). Tecnología y conflicto: Historia de la tractorización en México, En: SEMIHAAA. Memoria PIHAAA/ CIESTAAMX Seminario de Resultados de Investigación. Universidad Autónoma de Chapingo, Centro de Investigaciones Económicas, Sociales y Tecnológicas de la Agroindustria y la Agricultura Mundial (CIESTAAM).

Palacios, R. M. I, Reyes, R. y Teodoro, M. (2003).SEMIHAAA Memoria PIHAAA/CIESTAAM XSEMINARIO de resultados de investigación. Universidad Autónoma de Chapingo, Centro de Investigaciones Económicas, Sociales y Tecnológicas de la Agroindustria y la Agricultura Mundial (CIESTAAM), Texcoco, México.

Palacios Rangel, M. I. y Ledesma J. O. (2012). Los tractores agrícolas de México. Revista Mexicana de Ciencias Agricolas, 4, 812-824.

Plan de Desarrollo Municipal Zinacantepec 2016-2018. Municipio de Zinacantepec. Unidad de Información, Planeación, Programación y Evaluación. Zinacantepec, México, marzo de 2016. Consultado el 15 de agosto de 2017. Disponible en https://www.zinacantepec.gob.mx/z_docs/normatividad/files/Plan\%20 de\%20Desarrollo\%20Municipal.pdf.

Ramírez, V. B. (2007). Tecnología e implementos agrícolas: Estudio longitudinal en una región campesina de Puebla, México. Universidad Autónoma Chapingo (UACH). Rev. Geogr. Agrícola, 38(1):55-70. 
Reina Castro, J. L. y Hetz, E. (2012). Análisis del parque de tractores en el Ecuador. República del Ecuador: Editorial Académica Española.

Reina Castro, J. L. C. (2004). Análisis del parque de tractores agrícolas en el Ecuador. Consultada el 11 de octubre 2017. Disponible en http:// 152.74.96.144: 8080/sdx/udec/tesis/2004/reina_j/html/index-frames.html acceso8enero2006.

SAGARPA (Secretaría de Agricultura, Ganadería, Desarrollo Rural, Pesca y Alimentación). (2011). Disponible en http://www.sagarpa.gob.mx/agricultura/info/sust/suelo/ind_mec.swf.

Sánchez Hernández, M. A., Ayala, G. A. V., Cervantes, O. A. V., Garay, H. R., De la O, O. M., Martínez, T. M. y Guillermina, V L. N. (2014). Diagnóstico de la maquinaria agrícola en Amecameca y Texcoco, Estado de México. Agricultura, Sociedad y Desarrollo, 11(4). Consultado el 10 de abril de 2018. Disponible en http://www.scielo.org.mx/scielo.php?script=sci_arttext\&pid=S1870-54722014000400004

Selivanov, A. I. (1972.) Fundamentos de la teoría de envejecimiento de la maquinaria. Moscú: MIR.

Soto, M. (1983). Introducción al estudio de la maquinaria agrícola. México: Trillas.

Vaca García V. M., Martínez, V. J. J., González, H. A., Morales, R. E. J., Zamudio, G. B. y Gutiérrez, R. F. (2014). Compactación de un vertisol bajo tres sistemas de labranza en maíz (Zea Mays L.), Revista Mexicana de Ciencias Agricolas, 5(8).

\section{BY-NC-ND}

